\title{
Correction: Number density distribution of solvent molecules on a substrate: a transform theory for atomic force microscopy
}

Cite this: Phys. Chem. Chem. Phys. 2016, 18, 19973

DOI: $10.1039 /$ c6cp90175a

www.rsc.org/pccp

\author{
Ken-ichi Amano, ${ }^{\text {a }}$ Yunfeng Liang, ${ }^{\text {b }}$ Keisuke Miyazawa, ${ }^{c}$ Kazuya Kobayashi, ${ }^{\text {ab }}$ \\ Kota Hashimoto, ${ }^{a}$ Kazuhiro Fukami, ${ }^{d}$ Naoya Nishi, ${ }^{a}$ Tetsuo Sakka, ${ }^{a}$ Hiroshi Onishi ${ }^{e}$ \\ and Takeshi Fukumac
}

Correction for 'Number density distribution of solvent molecules on a substrate: a transform theory for atomic force microscopy' by Ken-ichi Amano et al., Phys. Chem. Chem. Phys., 2016, 18, 15534-15544.

The original Fig. 8 and 9 should be replaced with the revised versions below. The original Fig. 8 was not the short range force curve, but that of the total force. In the revised figure, the short range force curve is shown, which is obtained by subtracting the long range force curve from the total force curve. The revised Fig. 9 is the hydration structure on mica calculated from the revised Fig. 8. In addition, the values $1.0 \mathrm{~nm}$ written in page 15541 of the published article should be replaced by $0.5 \mathrm{~nm}$. Moreover, the following sentence written in page 15542 of the published article "In fact, the effective diameter of the real probe in this study has been estimated to be about $1.0 \mathrm{~nm}$, which is three to four times larger than that of a water molecule." should be replaced by "In fact, the effective diameter of the real probe in this study has been estimated to be about $0.5 \mathrm{~nm}$, which is about two times larger than that of a water molecule.".

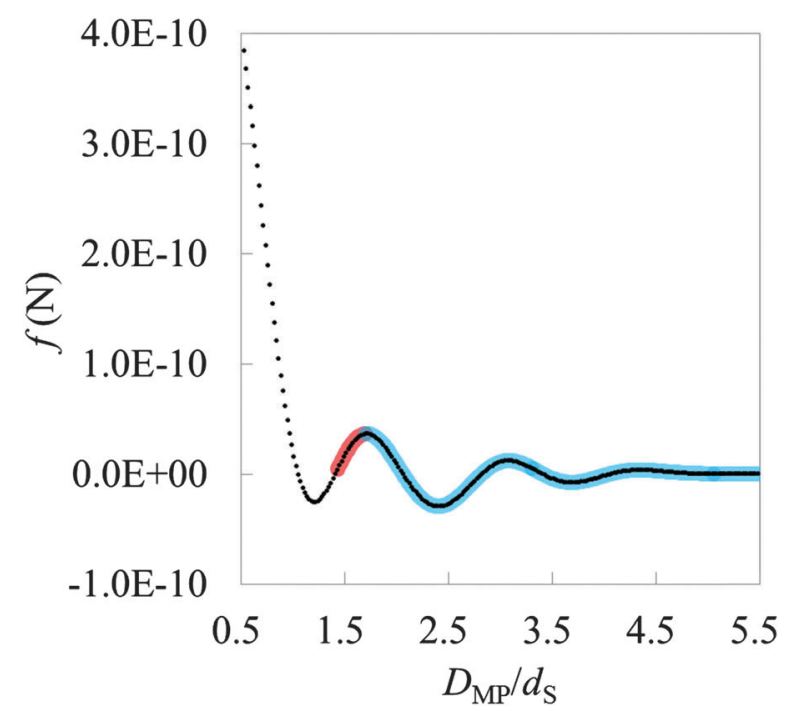

Fig. 8 The averaged short range force curve on mica surface measured by AFM, where $D_{M P}$ is the relative distance between the mica surface and the probe. On mica surface, such a typical shape can be measured. ${ }^{13,49}$ The plot represents the experimental data. The thick curve (pair of the red and blue curves) represents the fitted curve. The left and right sides of the first local maximum are colored red and blue, respectively. The blue thick curve is used for HPA transform.

\footnotetext{
${ }^{a}$ Department of Energy and Hydrocarbon Chemistry, Graduate School of Engineering, Kyoto University, Kyoto 615-8510, Japan. E-mail: amano.kenichi.8s@kyoto-u.ac.jp

${ }^{b}$ Environment and Resource System Engineering, Kyoto University, Kyoto 615-8540, Japan

${ }^{c}$ Division of Electrical Engineering and Computer Science, Kanazawa University, Kakuma-machi, Kanazawa 920-1192, Japan

${ }^{d}$ Department of Materials Science and Engineering, Graduate School of Engineering, Kyoto University, Kyoto 606-8501, Japan

${ }^{e}$ Department of Chemistry, School of Science, Kobe University, Nada, Kobe, Hyogo 657-8501, Japan
} 


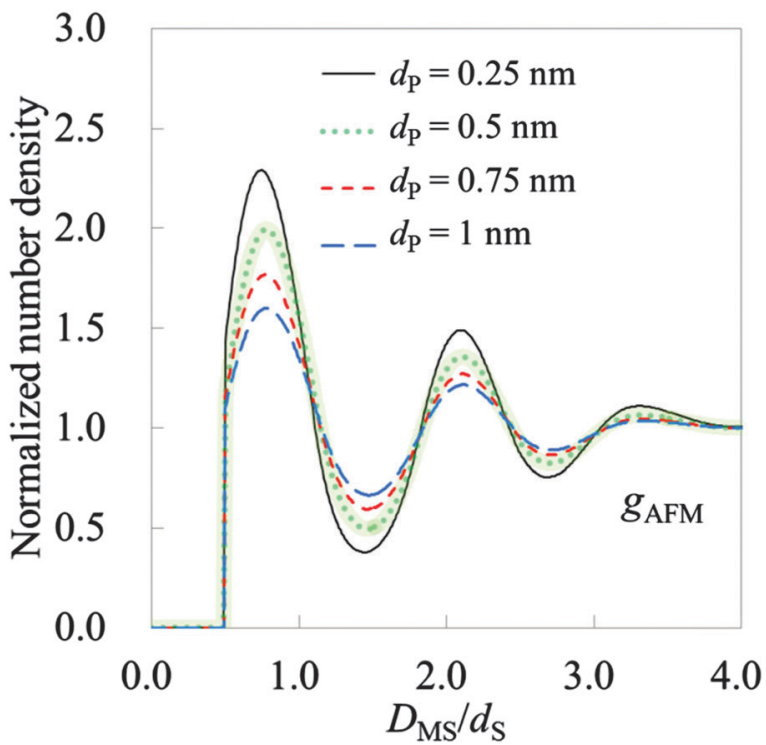

Fig. 9 Hydration structure on mica estimated by HPA transform with the patch method, where $D_{\text {MS }}$ is relative distance between the mica surface and the water molecule. The solid, dotted, short-dashed, and long-dashed lines represent $g_{\text {AFMS }}$ calculated with the effective diameters $0.25,0.5,0.75$, and $1 \mathrm{~nm}$, respectively. $g_{\text {AFM }}$ calculated with the $0.5 \mathrm{~nm}$ in the effective diameter is highlighted by a thick green line, because it is the most similar in amplitude to those obtained by $\mathrm{X}$-ray reflectivity, ${ }^{1} \mathrm{MD}^{53,54}$ and $M \mathrm{MC}^{55}$ simulations.

The Royal Society of Chemistry apologises for these errors and any consequent inconvenience to authors and readers. 